\title{
Tratamientos tópicos de los trastornos de la mucosa oral y la mucosa genital. Formulación magistral
}

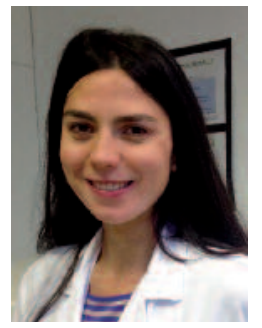

Lucía Alonso Pérez Farmacéutica responsable del área de Farmacotecnia. Servicio de Farmacia. Hospital Universitario 12 de Octubre. Madrid.

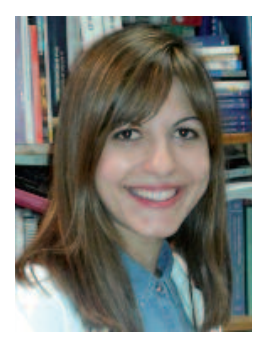

Carmen García Muñoz Farmacéutica responsable del Centro de Información de Medicamentos. Servicio de Farmacia. Hospital Universitario 12 de Octubre. Madrid.

\section{INTRODUCCIÓN}

La formulación magistral ha sido durante siglos una parte fundamental de la actividad profesional de los farmacéuticos y, hasta hace pocas décadas, la única vía a través de la que se elaboraban de forma apropiada los medicamentos para su uso medicinal. El desarrollo de la industria farmacéutica amplió notablemente las posibilidades de investigación y producción a gran escala de medicamentos, lo que ha supuesto un avance trascendental hacia una medicina más universalizada.

Sin embargo, existen pacientes que necesitan medicamentos adaptados a sus necesidades y que la industria no fabrica. La solución la ofrece la formulación magistral, que permite crear medicamentos individualizados.

En este artículo, nos vamos a centrar en el arsenal terapéutico que tenemos en el terreno de la formulación magistral para tratar trastornos de la mucosa oral y genital.

\section{EXCIPIENTES PARA}

\section{LA MUCOSA VAGINAL}

Para realizar la formulación magistral, hay que tener en cuenta la zona en la que se va a aplicar, las características que tiene el principio activo y el excipiente. Las formas farmacéu- ticas más utilizadas son los óvulos, las cremas y los geles. Los excipientes deben poseer alta tolerancia y bajo poder irritante y ser adecuados para la aplicación en la mucosa genital.

Los excipientes para realizar óvulos pueden ser:

1. Glicerogelatinas (hidromiscibles): generalmente, son de acción local, pero la mucosa vaginal puede, en casos específicos, servir de vía de reabsorción sistémica. Se utilizan para elaborar óvulos que contienen principios activos con efectos antiinflamatorios y antisépticos locales. Se debe evitar preparar óvulos con medicamentos muy activos, ya que podrían ocasionar intoxicaciones por absorción indeseada.

2. Polietilenglicoles (hidromiscibles): tienen buena estabilidad; químicamente son más reactivos que los excipientes grasos, no son irritantes ni tóxicos, y reducen la actividad de algunos antibióticos como la bacitracina y la penicilina.

3. Excipientes grasos: se utilizan para elaborar óvulos con liberación rápida del principio activo y de corta duración.

También se utilizan geles o cremas que no irriten la mucosa como excipientes para la formulación magistral. 


\section{EJEMPLOS DE FORMULACIÓN MAGISTRAL PARA LOS TRASTORNOS DE LA MUCOSA GENITAL}

\section{Vulvodinia}

Se define como un síndrome de malestar de la piel de la vulva caracterizado por síntomas como dolor intenso, prurito, quemazón, sensación punzante o de inflamación, irritación, picazón y dispareunia. Puede afectar a toda la zona o a algún punto concreto, ser persistente o intermitente, sin ninguna otra alteración clínica infecciosa o dermatológica ni de laboratorio que pueda explicar la molestia.

Varios estudios indican que, aproximadamente, el 10-16\% de las mujeres han tenido dolor vulvar crónico no explicado en algún momento de su vida. La vulvodinia aparece en mujeres caucásicas y africanas con una frecuencia similar, que puede ser mayor en mujeres de origen hispano ${ }^{1}$. Se desconoce la incidencia en asiáticas. Generalmente, aparece después de la adolescencia y hasta la novena década de la vida, con una media de edad de 36 años.

Se ha observado que la mayoría de mujeres que sufren vulvodinia presentan disfunción de los músculos del suelo pélvico ${ }^{2}$. Muchas mujeres con vulvodinia padecen dolores de cabeza crónicos, fibromialgia, estreñimiento crónico, así como aumento de la frecuencia urinaria ${ }^{3}$. Existe una implicación psicológica en todos los casos.

Además de las medidas generales —utilizar jabón íntimo para pieles sensibles, evitar irritantes, usar ropa interior de algodón y realizar ejercicios específicos para el suelo pélvico-, se recomiendan medicamentos para el dolor neuropático. En cuanto al tratamiento tópico, se recomiendan preparaciones de lidocaína al $5 \%$, amitriptilina al $2 \%$, doxepina al $5 \%$, gabapentina al 2-6\%, y óvulos de diazepam de 5-20 mg, capsaicina al 0,05\%, nitroglicerina, y amitriptilina al $2 \% /$ baclofeno al $2 \%{ }^{4}$.

\section{Fórmula magistral ${ }^{5}$}

- Doxepina al $5 \%$.

- Crema vaginal o emulsión no iónica: 30 g.
Fórmula magistral ${ }^{5}$

- Amitriptilina al $2 \%$.

- Crema acuosa de cetomacrogol: c.s.p. (cantidad suficiente para) $50 \mathrm{ml}$.

Fórmula magistral 5

- Gabapentina al $6 \%$.

- Crema vaginal: c.s.p. $50 \mathrm{ml}$.

\section{Liquen escleroso}

El liquen escleroso es una enfermedad dermatológica benigna, crónica y progresiva, caracterizada por una marcada inflamación y adelgazamiento epitelial. Aparece frecuentemente en la región anogenital. La etiología es desconocida y afecta de forma fundamental a mujeres posmenopaúsi$\operatorname{cas}^{6,7}$. Se estima una prevalencia de 1 de cada 3001000 individuos. Las manifestaciones clínicas más habituales son prurito, dolor e irritación. Los tratamientos más efectivos son los corticoides; también existen varias fórmulas magistrales para el liquen escleroso vulvar.

\section{Fórmula magistral ${ }^{5}$}

- Propionato de clobetasol al 0,05\%.

- Lidocaína al $2 \%$.

- Crema hidratante vaginal: c.s.p. 50 g.

Fórmula magistral 5

- Propionato de testosterona al 2,5\%.

- Excipiente graso hidromiscible: c.s.p. 100 g.

\section{EJEMPLOS DE FORMULACIÓN MAGISTRAL PARA LOS TRASTORNOS DE LA MUCOSA ORAL}

\section{Aftas}

La estomatitis aftosa o aftas bucales recurrentes es la causa más común de úlceras en la boca. Algunos factores que predisponen al desarrollo de estomatitis aftosa recurrente son la tendencia familiar, los factores hormonales y el estrés emocional. Las deficiencias de vitaminas y minerales 
Alonso Pérez L et al. Tratamientos tópicos de los trastornos de la mucosa oral y la mucosa genital. Formulación magistral

también se han relacionado con la patogenia, concretamente, el déficit de vitamina $\mathrm{B}_{12}$. Otras causas son el uso de antimetabolitos (metotrexato) y la neutropenia de cualquier causa ${ }^{8}$. Enfermedades como el síndrome de Behçet cursan con lesiones aftosas orogenitales y oftálmicas.

Actualmente, existen pocos medicamentos comercializados para el tratamiento de las afecciones de la mucosa oral, por lo que, en muchas ocasiones, debemos recurrir a la formulación magistral.

La mayor parte del epitelio bucal no se encuentra queratinizado, de modo que habrá mayor permeabilidad a las sustancias que apliquemos.

La humedad permanente existente en la mucosa oral hace necesario utilizar vehículos específi$\cos$. La adhesividad sobre la mucosa bucal a lo largo del tiempo es fundamental para que los principios activos manifiesten una acción progresiva y duradera. En función de esta adhesividad, existen distintos tipos de vehículos:

- Excipiente adhesivo oral: puede permanecer adherido a la mucosa desde 30 minutos a 2 horas, dependiendo del grosor de la capa aplicada y la motilidad bucal. Permite un mayor tiempo de contacto que los geles, una menor dosis total de principio activo que aplicar y una liberación más lenta del principio activo, debido a que combinan sustancias hidrófobas (vaselina, Plastibase) con gelificantes (carboximetilcelulosa, gelatina, pectina). Cuando se utilice este tipo de formulaciones, se recomienda secar la mucosa antes de la aplicación, no comer ni beber durante una hora y restringir el habla.

- Gel adhesivo oral: son geles formulados a partir de sorbitol, propilenglicol o sustancias gelificantes, como la carboximetilcelulosa sódica. Son una alternativa a los excipientes adhesivos en pacientes que tengan dificultad para aplicarlos o no toleren su textura grasa o su sabor. El tiempo de permanencia del principio activo en la formulaciones en gel es menor que el de los excipientes adhesivos.
- Colutorios: pueden emplearse cuando las lesiones son de tipo erosivo y se extienden en gran cantidad de la mucosa oral. Se puede formular en forma de solución acuosa o hidroalcohólica para enjuagues. Hay que intentar evitar usar alcohol en la formulación para no irritar las lesiones. Se incorporan productos suavizantes y extractos vegetales ricos en mucina y otros productos como el llantén. Es importante incorporar aromatizantes y saborizantes.

\section{Fórmulas magistrales ${ }^{9}$}

\section{Con glucocorticoide:}

- Clorhidrato de lidocaína al 2\% (puede variar entre el 1 y el $2 \%$ ).

- Acetónido de triamcinolona al 0,1\%.

- Vaselina líquida al $2 \%$.

- Excipiente adhesivo para mucosa bucal: c.s.p. $50 \mathrm{~g}$.

La función de la vaselina líquida en esta formulación es actuar como agente dispersante, que ayuda a la incorporación de los principios activos en la pomada adhesiva. Se recomienda que el agente dispersante sea de la misma polaridad que el principio activo (en este caso, hidrófobo). El propionato de clobetasol, el acetónido de triamcinolona y la hidrocortisona son liposolubles, mientras que la dexametasona 21-fosfato y el acetato de metilprednisolona son hidrosolubles ${ }^{10}$.

El dispersante debe añadirse en cantidad similar a la de los principios activos a dispersar. Si no se usa un dispersante o no es el adecuado, puede producirse un error de dosificación tras la aplicación de la pomada por el paciente por falta de homogeneización ${ }^{11}$.

\section{Con anestésico:}

- Lidocaína al 0,5-2\%.

- Carboximetilcelulosa al 1-1,5\%.

- Solución acuosa: c.s.p. $100 \mathrm{ml}$.

Puede emplearse como enjuague. Se puede utilizar antes de las comidas y para calmar el dolor. 
Se debe tener cautela en pacientes encamados, ya que puede suprimir el reflejo de la epiglotis, con el peligro de broncoaspiración.

\section{Lengua negra vellosa}

Afecta al dorso de la lengua y se caracteriza por la presencia de formaciones papilomatosas con coloración oscura de intensidad variable ${ }^{12}$. Se considera el tabaco como el primer factor etiopatogénico.

\section{Fórmula magistral}

- Urea al $40 \%$.

- Clotrimazol al $1 \%$.

- Ácido retinoico al 0,05-0,1\% (añadir o no en función del grado de hiperqueratosis).

- Gel hidroalcohólico: c.s.p. 50 g.

Cepillarse la lengua con el gel y un cepillo de dientes durante 1 o 2 minutos.

\section{Liquen plano oral}

Enfermedad inflamatoria de causa desconocida. Se caracteriza por la aparición de lesiones en la mucosa que reviste la cara interna de las mejillas, las encías o la lengua. Pueden ser lesiones blanquecinas, eritematosas o erosivas (ulceradas), que pueden ocasionar molestias que se acentúan con la ingesta de alimentos irritantes. Los corticoides tópicos son el tratamiento de primera elección ${ }^{13}$.

\section{Fórmula magistral}

- Ácido retinoico al 0,1\%.

- Propionato de clobetasol al 0,05\%.
- Clotrimazol al $1 \%$.

- Excipiente adhesivo oral: $40 \mathrm{~g}$.

\section{BIBLIOGRAFÍA}

1. Harlow BL, Stewart EG. A population-based assessment of chronic unexplained vulvar pain: have we underestimated the prevalence of vulvodynia? J Am Med Womens Assoc. 2003;58(2):82-8.

2. Hartmann D. Chronic vulvar pain from a physical therapy perspective. Dermatol Ther. 2010;23(5):505-13.

3. Reed B, Harlow S, Sen A, Edwards RM, Chen D, Haefner HK. Relationship between vulvodynia and chronic comorbid pain conditions. Obstet Gynecol. 2012;120(1):145-51.

4. Edwards L. Vulvodynia. Clin Obstet Gynecol. 2015;58(1):14352.

5. Bou Camps L, Suñer Ollé E. Formulación magistral en patología vulvo-vestibular. Barcelona: VIII Forum Aprofarm [sede web]; 2015. Disponible en: http://aprofarm.org/doc/9. PATOLOGIA_VULVO_VAGINAL.pdf

6. Guerra-Tapia A. Liquen escleroso. Actas Dermosifiliogr. 2003;94(10):633-41.

7. Guerra-Tapia A, González-Guerra E. Liquen escleroso de la vulva. Monogr Dermatol. 2005;28:184-91.

8. Goldstein BG, Goldstein AO. Oral lesions. UpToDate; 2015. Disponible en: http://www.uptodate.com/

9. Sánchez Palau L, Sáez Martín LC. Aftas mucosas. Barcelona: VI Forum Aprofarm [sede web]; 2013. Disponible en: http:// www.aprofarm.org/doc/11_Presentacion_AftasMucosas.pdf

10. Iglesias M, Codina A. Formulación en casos complejos de mucosa oral. Barcelona: IV Forum Aprofarm [sede web]; 2011. Disponible en: http://www.aprofarm.org/doc/02_Formulacion_ terapeutica_casos_dificiles_Mucosa_Oral_Dra_Iglesias.pdf

11. Acofarma. Incorporación de activos en excipiente adhesivo oral. Disponible en: http://www.formulacionmagistral.org/ blog/elaboracion/incorporacion-de-activos-en-excipienteadhesivo-oral/

12. Bosch García RJ. Tratamiento de la lengua negra vellosa. Piel. 2011;26(5):242-5.

13. González-Guerra E, Romero Aguilera G, Llamas Martín R, Guerra Tapia A. Liquen plano. En: Guerra Tapia A (ed.). Manual y atlas de las enfermedades de los genitales del varón. Barcelona: Editorial Glosa, S.L.; 2008. p. 179-84. 